\title{
QUADRATIC PROGRAMMING IN INSURANCE
}

\author{
H. Schmitter and E. Straub \\ Zürich
}

\section{ABStract AND Introduction}

Quadratic programming means maximizing or minimizing a quadratic function of one or more variables subject to linear restrictions i.e. linear equations and/or inequalities.

Among the numerous insurance problems which can be formulated as quadratic programs we shall only discuss four, namely the Credibility, Retention, IBNR and the Cost Distribution problems.

Generally, there is no explicite solution to quadratic optimization problems, only statements about the existence of a solution can be made or some algorithm may be recommended in order to get exact or approximate numerical solutions. Restricting ourselves to typical problems of the above mentioned type, however, enables us to give an explicit solution in terms of general formulae for quite a number of cases, such as the onedimensional credibility problem, the retention problem and--under relatively week assumptionsfor the IBNR-problem.

The results given here are by no means new. The only goal of this paper is to describe a few fundamental insurance problems from a common mathematical standpoint, namely that of quadratic programming and at the same time, to draw attention to a few special aspects and open questions in this field.

\section{The Credibility Problem}

We consider a portfolio consisting of $N$ different risk categories $j(j=1,2, \ldots N)$ for each of which claims statistics over the last $n$ years $(i=1,2, \ldots \ldots n)$ are available. With $P_{i j}>0$, we denote the volume of class no. $j$ for the year no. $i$ (volume $=$ number of risks, total sum insured or underlying premium volume) and with $Y_{i j}$ the corresponding total of claims (or number of claims) so that the yearly loss ratios (or the claims frequency) are given by $X_{i j}=Y_{i j} / P_{i j}$. 
For the entire statistical period we therefore have

$$
\text { a volume } P_{\cdot j}=\sum_{i=1}^{n} P_{i j}
$$

and a loss ratio $X_{\cdot j}=Y_{\cdot j} / P_{\cdot j}$ with $Y_{\cdot j}=\sum_{i=1}^{n} Y_{i j}$.

Now, the so-called credibility problem consists of estimating the expected value of $X{ }_{k}$ for a fixed risk category $k$ under the condition that the latter depends on a risk parameter $\theta$ which characterizes the heterogeneity of the portfolio. Or, expressed a little more mathematically:

Estimate $E\left[X \cdot_{k} \mid P_{\cdot k}=P, \theta_{k}=\theta\right]$

if $E\left[X{ }_{k} \mid P ._{k}=P, \theta_{k}=\theta\right]=\mu(\theta)$ independently of $P$,

$\operatorname{Var}\left[X \cdot_{k} \mid P_{\cdot k}=P, \theta_{k}=\theta\right]=\frac{\sigma^{2}(\theta)}{P}$

where $X_{{ }_{k}}$ and $X{ }_{\cdot j}$ are assumed to be stochastically independent for $j \neq k$ and fixed $\theta_{k}$ and $\theta_{j}$ and furthermore $\theta_{j}$ independent and identically distributed according to a distribution $U(\theta)$ which is called "structure function".

Confining ourselves to linear and unbiased minimum square estimates we may finally write:

For fixed $k$ determine $\alpha_{i j}(i=\mathrm{I}, 2, \ldots n ; j=\mathrm{I}, 2, \ldots N)$ such that

(i) $E\left[\left\{\mu\left(\theta_{k}\right)-\sum_{j=1}^{N} \sum_{i=1}^{n} \alpha_{i j} X_{i j}\right\}^{2}\right]=$ minimum

(ii). $E\left[\sum_{j=1}^{N} \sum_{i=1}^{n} \alpha_{i j} X_{i j}\right]=E_{\theta}[\mu(\theta)] \quad$ (unbiasedness)

(iii) $0 \leqslant \alpha_{i j} \leqslant \mathrm{I}$ for $i=\mathrm{I}, 2, \ldots n$ and $j=\mathrm{I}, 2, \ldots N$.

This is a quadratic program for the $n N$ unknowns $\alpha_{i j}$.

Its explicit solution is given by [2]

$$
\hat{\mu}_{k}=\sum_{j=1}^{N} \sum_{i=1}^{n} \alpha_{i j} X_{i j}=\gamma_{k} X ._{k}+\left(\mathrm{I}-\gamma_{k}\right) \bar{X}
$$

where $\gamma_{k}=\frac{w P \cdot k}{v+w P \cdot k}=$ Credibility of risk category $k$ 
with $v=E_{0}\left[\sigma^{2}(\theta)\right], w=\operatorname{Var}_{\theta}[\mu(\theta)]$

and $\bar{X}=\sum_{j=1}^{N}\left(\gamma_{j} / \gamma\right) X \cdot j \quad$ with $\gamma=\sum_{j=1}^{N} \gamma \cdot j$.

We can therefore say that the estimate which is optimal in the above sense is a weighted average of the individual claims experience $X{ }_{k}$ and the overall claims experience $\vec{X}$. The latter is in general, however, not identical with the "natural" portfolio average $X$. where the individual claims experiences are weighted with the relative premium volumes, namely

$$
X \ldots=\sum_{j-1}^{N}(P \cdot j / P \ldots) X \cdot j \quad \text { with } P \ldots=\sum_{j=1}^{N} P_{\cdot j}
$$

but instead, the correct weights are, as we have just seen, the relative credibilities, since

$$
X=\sum_{j=1}^{N}(\gamma \cdot j / \gamma) X_{\cdot j} \quad \text { with } \quad \gamma=\sum_{j=1}^{N} \gamma \cdot j
$$

From the special form of the credibilities $\gamma \cdot j$ we can also immediately see that

- the larger the risk category $j$ (i.e. the larger $P \cdot j$ ), the larger is $\gamma \cdot j$

- the larger the variations of the $X_{i j}$ 's in time (i.e. the larger $v$ ), the smaller is $\gamma \cdot j$

- the larger the variations of the $X_{i j}$ 's within the portfolio (i.e. the larger $w$ ), the larger is $\gamma \cdot j$

These observations match perfectly with what we may already have intuitively expected and this makes it relatively easy to discuss the general results also with insurance practitioners who are not necessarily mathematically oriented.

However, this very practical advantage seems to be lost as soon as we change to two- or more-dimensional risk parameters, since at least so far, we have not been able to write up a similar kind of explicit solution for $\theta=\left(\theta_{I}, \theta_{I I}\right)$, i.e. for the case where the portfolio is divided into subgroups by two or more criteria at the same time. 


\section{Retention Problems}

The retention in reinsurance arrangements can be determined by means of quadratic optimization as is demonstrated in the following summary of a chapter from [I].

Let us consider a portfolio consisting of $N$ independent risks, where the $i$-th risk is characterized by $S^{(i)}$, the accumulated claim in a given time interval. An individual reinsurance arrangement is a function $g_{i}$ which determines a retained portion $g_{i}\left[S^{(i)}\right]$ of $S^{(i)}$.

Confining ourselves to the proportional case, let $P^{(i)}$ denote the price demanded by the reinsurer for taking over the risk completely, and $P^{(i)} \cdot\left(\mathrm{I}-a_{i}\right)$ for accepting the cession $\mathrm{I}-a_{i}$. Then the stochastic variable

$$
Z=\sum_{i=1}^{N} a_{i}\left(P^{(i)}-S^{(i)}\right)
$$

measures the profit earned on the retained portion of the portfolio. Then our problem is that of finding those reinsurance arrangements which guarantee the given expected profit $E(Z)$ in the retention with the smallest possible deviations. In other words, we determine the $a_{i}$ so that

$$
\operatorname{Var}(Z)=\min
$$

under the additional condition that

$$
E(Z)=\text { constant. }
$$

For this purpose we introduce the Lagrange multiplier $\lambda$ and differenciate the function

$$
\phi=\operatorname{Var}(Z)+\lambda E(Z)
$$

partially with respect to $a_{i}$. Because of the independence of the $S^{(i)}$ we have

and

$$
\operatorname{Var}(Z)=\sum_{i=1}^{N} a_{i}^{2} \operatorname{Var}\left(S^{(i)}\right)
$$

From

$$
E(Z)=\sum_{i=1}^{N} a_{i}\left(P^{(i)}-E\left[S^{(i)}\right]\right)
$$

$$
\left.\frac{\partial \phi}{\partial a_{j}}=2 a_{j} \operatorname{Var}\left[S^{(j)}\right]+\lambda\left(P^{(j)}-E^{r} S^{(j)}\right]\right)=0
$$


for all $j$ it follows that

$$
a_{j}=C \frac{P(j)-E\left[S^{(j)}\right]}{\operatorname{Var}\left[S^{(j)}\right]}
$$

$C$ is called "absolute Retention" (cf. [I]).

It depends on the insurance carrier's stability policy. If e.g. according to the ruin criterion, the probability of ruin should be less than a given $P_{0}$ it is shown in $[\mathrm{I}]$ that

$$
C=\frac{2 u}{\rho \cdot\left|\ln P_{0}\right|}
$$

under the assumption that $S^{(j)}=\sum_{i=1}^{A_{j}} Y_{i}^{(j)}$

where

$A_{j}$ is a Poisson distributed number of claims variable,

$Y_{i}^{(j)}$ are independent non-negative variables with identical distribution,

$u$ is the amount of free reserves, and

$p$ is the ratio of proportional loading in the retained portion in relation to that in reinsurance.

Replacing those $a_{j}$ which exceed I by I yields an optimal solution for a smaller $E(Z)$ than the one given.

\section{The Ibnr-Problem}

In this context IBNR stands for both "Incurred But Not Reported" and inadequate reserving of already reported claims. It is a wellknown fact when dealing with so-called "longtail business" that the final number of claims and final total yearly claims cost are only known after several years. The insurer-and especially the excess of loss reinsurer of Motor Liability e.g.-is therefore forced to estimate final loss ratios either for premium calculation or reserving purposes on the basis of incomplete statistics. These purely statistical observations are usually presented as follows in what is called an "IBNR-triangle": 


\begin{tabular}{ccccccc}
\hline $\begin{array}{l}\text { year of } \\
\text { occurrence }\end{array}$ & Loss ratios as per the end of \\
\hline$I 966$ & $\mathrm{i}=5$ & $X_{5}(1)$ & $X_{5}^{(2)}$ & $X_{5}^{(3)}$ & $X_{5}^{(4)}$ & $X_{5}^{(5)}$ \\
$I 967 \quad \mathrm{i}=4$ & & $X_{4}^{(1)}$ & $X_{4}^{(2)}$ & $X_{4}^{(3)}$ & $X_{4}^{(1)}$ \\
$I 968$ & $\mathrm{i}=3$ & & & $X_{3}^{(1)}$ & $X_{3}^{(2)}$ & $X_{3}^{(3)}$ \\
$I 969 \quad \mathrm{i}=2$ & & & & $X_{2}^{(1)}$ & $X_{2}^{(2)}$ \\
$I 970 \quad \mathrm{i}=\mathrm{I}$ & & & & & $X_{1}^{(1)}$ \\
\hline
\end{tabular}

e.g. $X_{4}^{(3)}=$ loss ratio for 1967 as per December 3 1st, 1969

If there are $m$ statistical years in total, we have a triangle

$$
\nabla X=\left\{X_{i}^{(h)} \mid i=\mathrm{I}, 2, \ldots . m ; h=\mathrm{I}, 2, \ldots . i\right\}
$$

and our problem is to estimate the final loss ratio e.g. for the year $i=q$ or, in other words:

For the conditional expectation $E\left[X_{q}^{(\infty)} \mid \nabla X\right]$ determine an estimator $\hat{\mu}_{q}^{(\infty)}$ such that

and

$$
E\left[\left\{E\left[X_{q}^{(\infty)} \mid \nabla X\right]-\hat{\mu}_{q}^{(\infty)}\right\}^{2}\right]=\text { minimum }
$$

$$
E\left[\hat{\mu}_{q}^{(\infty)}\right]=E\left[E\left[X_{q}^{(\infty)} \mid \nabla X\right]\right] \text {. }
$$

We are thus looking for an unbiased minimum square estimator for the-at present unknown-final loss ratio of the year $i=q$.

Under the assumptions

(i) $X_{i}^{(h)}$ stochastically independent of $X_{i^{\prime}}^{\left(h^{\prime}\right)}$ for $i \neq i^{\prime}$

(ii) $E\left[X_{i}^{(h)}\right]=e^{(h)}$ independently of $i$

(iii) $P_{i} \operatorname{Cov}\left[X_{i}^{(h)}, X_{i}^{\left(h^{\prime}\right)}\right]=c_{h h^{\prime}}$ independently of $i$ and with $P_{i}=$ underlying premium volume of year $i$

(iv) $\hat{\mu}_{q}^{(\infty)}=\sum_{i=1}^{n} \sum_{h-1}^{i} \alpha_{i h} X_{i}^{(h)}$ i.e. we confine ourselves to linear estimators

(v) loss ratio already final after $m$ years: $X_{i}^{(m)} \equiv X_{i}^{(\infty)}$

we are again confronted with a quadratic program, this time for the $\frac{m(m+\mathrm{I})}{2}$ unknowns $\alpha_{i n}$ and subject to simply one boundary condition, namely

$$
e^{(m)}=\sum_{i=1}^{m} \sum_{h=1}^{i} \alpha_{i h} e^{(h)}
$$


According to [3] the general solution of this problem is described by the two following equations

$$
\begin{aligned}
& \hat{\mu}_{q}^{(m)}=\alpha \sum_{i=1}^{m} P_{i}\left(\hat{\mathrm{e}}_{i}, \beta_{i}^{1} \underline{X}_{i}\right)+\left(\underline{c}_{m q}, \beta_{q}^{-1} \underline{X}_{q}\right) \\
& e^{(m)}=\alpha \sum_{i=1}^{m} P_{i}\left(\underline{e}_{i}, \beta_{i}^{-1} \underline{e}_{i}\right)+\left(\underline{c}_{m q}, \beta_{q}^{-1} \underline{e}_{q}\right)
\end{aligned}
$$

where the following vector and matrix notation has been used

$$
e_{i}=\left[\begin{array}{c}
e^{(1)} \\
e^{(2)} \\
\cdot \\
\cdot \\
\cdot \\
\cdot \\
e^{(i)}
\end{array}\right], \beta_{i}=\left[\begin{array}{cccc}
c_{11} & c_{12} & \ldots & c_{1 i} \\
c_{21} & c_{22} & \ldots & c_{2 i} \\
\cdot & & \\
\cdot & & \\
\cdot & & \\
\cdot & & & \\
c_{i 1} & c_{i 2} & \ldots & c_{i i}
\end{array}\right], X_{i}=\left[\begin{array}{c}
X^{(1)} \\
X_{i}^{(2)} \\
\cdot \\
\cdot \\
\cdot \\
\cdot \\
X_{i}^{(i)}
\end{array}\right], c_{m i}=\left[\begin{array}{l}
c_{m 1} \\
c_{m 2} \\
\cdot \\
\cdot \\
\cdot \\
\cdot \\
c_{m i}
\end{array}\right]
$$

$\left(\beta_{i}^{-1}=\right.$ inverse of the covariance matrix $\left.\beta_{i}\right)$ and where $(a, b)$ denotes the inner product of the two vectors $a$ and $b$.

If the expectations $e_{m}$ and covariances $\beta_{m}$ are known, we can therefore first calculate the multiplier $\alpha$ from equation (2) and afterwards, by introducing $\alpha$ into (x), directly get the estimator $\hat{\mu}_{q}^{(m)}$. Equation (2) is, by the way, nothing else than the condition of unbiasedness applied to equation (I).

As shown in [5], these calculations become much more transparent if

either (i) $X^{(h)}=X^{(h-1)}+Y^{(h)}, Y^{(h)}$ independent of $X^{(h-1)}(a d-$ ditive model)

or (ii) $X^{(h)}=\Lambda^{(h)} X^{(h-1)}, \Lambda^{(h)}$ independent of $X^{(h-1)}$ (multiplicative model)

or (iii) $X^{(h)}=\Lambda^{(h)} X^{(h-1)}+Y^{(h)} ; X^{(h-1)}, Y^{(h)}, \Lambda^{(h)}$ independent (the "mixed" model)

because in these cases the inverses $\beta^{-1}$ can all be explicitly written up (their elements are all equal to zero except those in the diagonal and in the two adjacent "diagonals"). 
When dealing with concrete practical problems, the parameters $e_{m}$ and $\beta_{m}$ are of course unknown, these parameters also have to be estimated from the information contained in the triangle $\nabla X$, a problem which-depending on one's view-may be formulated as yet another quadratic programme. In this context, the estimation of the covariances is of primary interest. Starting e.g. with estimators $\hat{c}_{h k}$ of the form

$$
\hat{c}_{h k}=\frac{\mathrm{I}}{m-h}\left\{\sum_{i=h}^{m} P_{i} X_{i}^{(h)} X_{i}^{(k)}-\left(\sum_{i=h}^{1 / m} P_{i}\right) \sum_{i=h}^{m} P_{i} X_{i}^{(h)} \sum_{i=h}^{m} P_{i} X_{i}^{(k)}\right\}
$$

we may perhaps discover that the corresponding estimators for the correlation coefficient, namely

$$
\hat{\rho}_{h k}=\frac{\hat{c}_{h k}}{\sqrt{\hat{c}_{h k} k}=}
$$

are neither bounded by $-I$ or $+I$ nor -as it seems reasonable to assume-monotonically increasing in $h$ for $h \leqslant k$. It is then indicated to "isotonize" the above $\hat{\rho}_{h k}$ values which is essentially equivalent to solving a specific quadratic program [4].

\section{The Cost Distribution Problem}

We consider an insurance portfolio divided with respect to two criteria, e.g. branches $i(i=\mathrm{I}, 2, \ldots, n)$ and countries $j(j=\mathrm{I}$, $2, \ldots, m)$, which has to contribute an amount $C$ to a fund, e.g. a catastrophy fund.

Furthermore, let $A_{1}, A_{2}, \ldots, A_{n}$ and $B_{1}, B_{2}, \ldots, B_{m}$ denote the contributions per branch and country, respectively. We assume

$$
\sum_{i=1}^{n} A_{i}=\sum_{j=1}^{m} B_{j}=C
$$

The following table represents the distribution of these costs, where $x_{i j} P_{i j}$ stands for the contribution of branch $i$ in country ${ }_{j}$, $P_{i j}$ being the corresponding volume (e.g. number of risks, sum insured or underlying premium volume). 


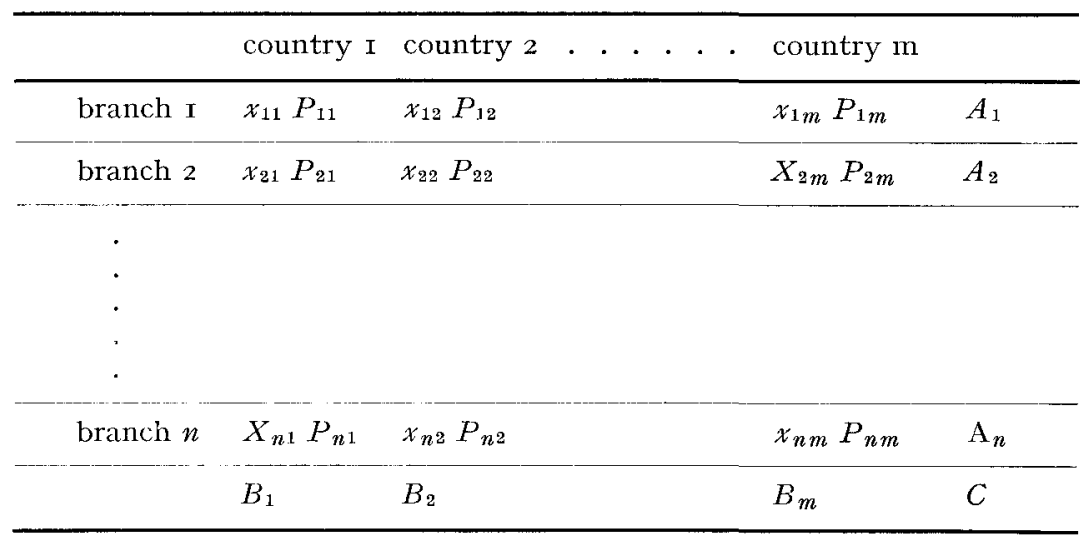

We write $A_{i}=a_{i} P_{i}, B_{j}=b_{j} P \cdot j, C=c P$.

where

$$
P_{i .}=\sum_{j=1}^{m} P_{i j}, P_{\cdot j}=\sum_{i=1}^{n} P_{i j} \text { and } P . .=\sum_{i=1}^{n} \sum_{j=1}^{m} P_{i j}
$$

Now our problem consists in minimizing the function

$$
\begin{array}{ll}
\sum_{i=1}^{n} \sum_{j=1}^{m}\left(x_{i j}-c\right)^{2} P_{i j} \quad \text { such that } \\
\sum_{j=1}^{m} x_{r j} P_{r j}=a_{r} P_{r} . & (r=\mathrm{I}, 2, \ldots \ldots, n) \\
\sum_{i=1}^{n} x_{i s} P_{i s}=b_{s} P . s & (s=\mathrm{I}, 2, \ldots, m) \\
x_{r s} \geqslant 0 . &
\end{array}
$$

As far as we know, this problem cannot be solved explicitly. We are thus forced to confine ourselves to a few remarks about the rare results found until now. First, treating the problem without any sign-restrictions, we form the Lagrangian

$$
\begin{gathered}
\phi=\sum_{j=1}^{m} \sum_{i=1}^{n}\left(x_{i j}-c\right)^{2} P_{i j}+2 \sum_{i=1}^{m} \theta_{i}\left(\sum_{j=1}^{n} x_{i j} P_{i j}-A_{i}\right) \\
+2 \sum_{j=1}^{m} \lambda_{j}\left(\sum_{i=1}^{n} x_{i j} P_{i j}-B_{j}\right) .
\end{gathered}
$$

Putting the partial derivatives with respect to $x_{r s}$ to o yields

$$
x_{r s}=c-\theta_{r}-\lambda_{s} .
$$


Summing up over $r$ and $s$, respectively, we get for the Lagrange multipliers $\theta_{r}$ and $\lambda_{s}$ the equations

$$
\begin{array}{ll}
\sum_{i=1}^{n} \theta_{i} P_{i s}+\lambda_{s} P \cdot s=\left(c-b_{s}\right) P_{s} \quad & (s=\mathrm{I}, 2, \ldots, m) \\
\theta_{r} P_{r}+\sum_{j=1}^{m} \lambda_{j} P_{r j}=\left(c-a_{r}\right) P_{r} . & (r=\mathrm{I}, 2, \ldots, n)
\end{array}
$$

which solved for $\theta$ yield

$$
\begin{array}{cr}
\theta_{r} P_{r}-\sum_{i=1}^{n} \theta_{i} \alpha_{r i}=\delta_{r} & (r=\mathrm{I}, 2, \ldots, n) \\
\text { with } \alpha_{r i}=\sum_{i=1}^{m} \frac{P_{r j} P_{i j}}{P_{\cdot j}} & \text { and } \delta_{r}=\sum_{j=1}^{m} b_{j} P_{r j}-A_{r}
\end{array}
$$

or in matrix-notation

$$
A \theta=\delta
$$

where

$$
a_{i i}=\sum_{\substack{k=1 \\ k \neq i}}^{n} \alpha_{i k}, a_{i k}=a_{k i}=-\alpha_{i k}
$$

and

$$
\sum_{i=1}^{n} \delta_{i}=0
$$

The rank of $A$ is less or equal to $n-\mathrm{I}$. In the sequel we assume rank $A=n-\mathrm{I}$. In this case the one-dimensional subspace $\underline{\theta}=p \cdot \underline{I}$ is the solution of the homogenous system $A \underline{\theta}=0$.

To find a special solution $\theta^{*}$ of the inhomogenous system we add the equation

$$
\theta_{1}^{*}=0
$$

because of $\sum_{i=1}^{n} \delta_{i}=0$ the system

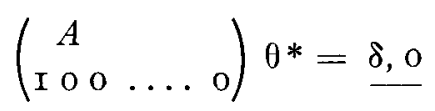

has at least one solution too. 
The solution of the system

$$
\left[\begin{array}{cccc}
\sum_{\substack{i=1 \\
i \neq 3}}^{n} \alpha_{2 i}-\alpha_{23} & -\alpha_{24} & \ldots & -\alpha_{2 n} \\
-\alpha_{23} & \sum_{\substack{i=1 \\
i \neq 3}}^{n} \alpha_{3 i} & -\alpha_{34} & -\alpha_{3 n} \\
-\alpha_{24}-\alpha_{34} & & \\
\cdot & \cdot & \\
\cdot & & \\
-\alpha_{2 n} & & \sum_{i=1}^{n-1} \alpha_{n i}
\end{array}\right]\left[\begin{array}{c}
\theta_{2}^{*} \\
\theta_{3}^{*} \\
\cdot \\
\cdot \\
\theta_{n}^{*}
\end{array}\right]=\left[\begin{array}{c}
\delta_{2} \\
\cdot \\
\delta_{3} \\
\cdot \\
\cdot \\
\delta_{n}
\end{array}\right]
$$

introduced in 5) and 4) yield the $x_{r s}$.

Explicit expressions become very complicated for $n>3$.

Yet, there are some rules for the computation of the determinant $\Delta$ of the matrix in 7): It is the sum of all products $\alpha_{i_{2}} k_{2} \alpha_{i_{3}} k_{3}$ $\ldots \alpha_{i_{n} i_{n}}$ in which every subscript appears at least once and in which no $h$ factors $(h=2,3, \ldots, n)$ may be ordered in a cycle as $\alpha_{i_{1} i_{2}} \alpha_{i_{2} i_{3}} \ldots \ldots \alpha_{i_{h} i_{h}}$.

Similar rules exist for the next lower subdeterminants of $\Delta$.

As an example in the case of $n=3$, we have $\Delta=\alpha_{12} \alpha_{13}+\alpha_{12} \alpha_{23}+\alpha_{13} \alpha_{23}$ and the solutions of 5) are

$$
\begin{aligned}
& x_{1 s}=b_{s}-\frac{\alpha_{23}}{\Delta} \frac{P_{2 s}+P_{3 s}}{P \cdot s} \delta_{1}+\frac{\alpha_{13}}{\Delta} \frac{P_{2 s}}{P \cdot s} \delta_{2}+\frac{\alpha_{12}}{\Delta} \frac{P_{3 s}}{P \cdot s} \delta_{3} \\
& x_{2 s}=b_{s}+\frac{\alpha_{23}}{\Delta} \frac{P_{1 s}}{P \cdot s} \delta_{1}-\frac{\alpha_{13}}{\Delta} \frac{P_{1 s}+P_{3 s}}{P \cdot s} \delta_{2}+\frac{\alpha_{13}}{\Delta} \frac{P_{3 s}}{P \cdot s} \delta_{3} \\
& x_{3 s}=b_{s}+\frac{\alpha_{23}}{\Delta} \frac{P_{1 s}}{P \cdot s} \delta_{1}+\frac{\alpha_{13}}{\Delta} \frac{P_{23}}{P \cdot s} \delta_{2}-\frac{\alpha_{12}}{\Delta} \frac{P_{1 s}+P_{2 s}}{P \cdot s} \delta_{3} .
\end{aligned}
$$

As the problem can be solved explicitly for $n=2$, presumably even with the sign restrictions, one is encouraged to try to solve the problem "iteratively" in a first step by distinguishing the first line of $A$, combining the second, third a.s.o. to one single line and solving this $2 x m$ problem ( 2 lines, $m$ columns), then in a second step dis- 
tinguishing the originally second line, combining the third to last a.s.o.

It seems, however, that this procedure is not generally applicable, at least the numerical examples we have dealt with so far by this method led, in some cases, to solutions, in others to contradictions.

\section{REFERENCES}

[I] H. Bühlmann, Mathematical Methods in Risk Theory, Springer Verlag, I970.

[2] H. Bühlmann und E. Straub, Glaubwürdigkeit für Schadensätze, Mitteilungen der Vereinigung Schweizerischer Versicherungsmathematiker, I970.

[3] E. Straud, On the Calculation of IBNR-Reserves, IBNR-The Price Winning Papers in the Boleslaw Monic Fund Competition 1971, Nederlandse Reassurantie Groep N.V. Amsrerdam 1972.

[4] R. Barlow, D. Bartholomew, G. Bremner and D. Brunk, Statistical Inference under Order Restrictions, John Wiley 1972.

[5] H. Kramreiter und E. Straub, On the Calculation of IBNR-Rescrves II, for publication in "Mitteilungen der Schweizerischen Versicherungsmathematiker" (autumn 1973). 